\title{
Prävention zeigt Erfolg: Verbesserung in allen Altersgruppen
}

Im internationalen Vergleich setzt Deutschland bei der Mundgesundheit Maßstäbe: In allen Altersgruppen zeigt sich ein deutlicher Kariesrückgang. Die Zahl der Parodontalerkrankungen nimmt zwar ab, der Behandlungsbedarf wird in Zukunft jedoch weiter steigen. Dies sind die zentralen Ergebnisse der seit Langem erwarteten fünften Deutschen Mundgesundheitsstudie (DMS V) des Instituts der Deutschen Zahnärzte (IDZ) im Auftrag von Bundeszahnärztekammer (BZ̈̈K) und Kassenzahnärztlicher Bundesvereinigung (KZBV), die in Berlin vorgestellt wurde.

Laut Studie hat die Verbreitung von Karies in allen vier befragten Altersgruppen abgenommen. Im Vergleich zu den Anfängen der DMS 1989 in Westdeutschland und 1992 in den neuen Ländern ging Karies bei den 12-jährigen Kindern um 90 Prozent zurück. Die aktuelle DMS V kommt zu dem Ergebnis, dass heute insgesamt 81 Prozent der Kinder kariesfrei sind. Bei jüngeren Erwachsenen zwischen 35 und 44 Jahren sind fast fünf Zähne weniger mit Karies belastet als noch vor knapp zwanzig Jahren. Bei den 65- bis 74-jährigen jüngeren Senioren nahm die Karieserfahrung der DMS zufolge in den vergangenen zwei Jahrzehnten sogar um sechs Zähne ab. „Die hervorragenden Werte bei Karies sind ein Beweis, dass die zahnmedizinische Prävention funktioniert“, stellte der Präsident der Bundeszahnärztekammer, Dr. Peter Engel, fest.

\section{Prävention und strukturierte Nachsorge}

Auch die Zahl der Parodontalerkrankungen nehme in der Bundesrepublik immer weiter ab. Allerdings leide dennoch mehr als die Hälfte der Erwachsenen an einer behandlungsbedürftigen parodontalen Erkrankung, acht Prozent sogar unter einer schweren Parodontitis. „Wir gehen davon aus, dass die acht Prozent schweren Parodontalerkrankungen eher zurückhaltend geschätzt sind und die tatsächliche Verbreitung in der Bevölkerung bis zu 14 Prozent beträgt“, erklärte Dr. A. Rainer Jordan, wissenschaftlicher Direktor des Instituts der Deutschen Zahnärzte (IDZ). Die demografische Entwicklung in Deutschland deute zudem darauf hin, dass in Fragen von parodondalen Erkrankungen mit einem deutlich steigenden Behandlungsbedarf $\mathrm{zu}$ rechnen sei. In diesem Zusammenhang betonte der KZBVVorstandsvorsitzende Dr. Wolfgang Eßer vor allem den bedeutenden Stellenwert der sprechenden Zahnheilkunde. „Diese muss Einfluss in den Leistungskatalog der gesetzlichen Krankenkassen finden und auch angemessen honoriert werden", forderte der KZBV-Chef.

Zusätzlich zur Prävention komme es bei parodontalen Erkrankungen auch auf eine strukturierte Nachsorge, die unterstützende Parodontitistherapie (UPT), an. „Hier könnte aus Sicht der Vertragszahnärzteschaft ein Bonusmodell greifen ähnlich wie schon bei der Versorgung mit Zahnersatz“, erklärte Eßer. Zusätzlich müsse die flächendeckende Aufklärung zu Parodontalerkrankungen geschärft werden.

\section{Schlechtere Mundgesundheit bei Pflegebedürftigen}

In der erstmals untersuchten Altersgruppe der älteren Senioren zwischen 75 und 100 Jahren deckt die DMS V signifikante Unterschiede in der Zahn- und Mundgesundheit zwischen Pflegebedürftigen und der gesamten Altersgruppe auf. Demnach ha-

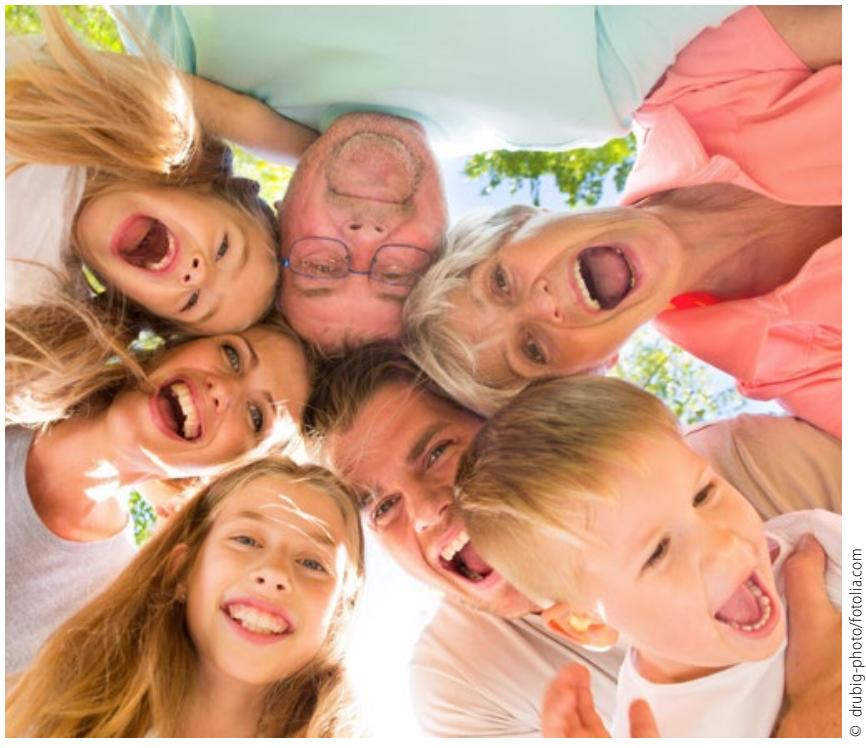

ben ältere Menschen mit Pflegebedarf eine höhere Karieserfahrung, weniger eigene Zähne und häufiger einen herausnehmbaren Zahnersatz als alle untersuchten Personen in der Altersgruppe. Zudem seien Pflegebedürftige häufiger auf Unterstützung bei der Mundhygiene angewiesen. Rund 60 Prozent seien nicht mehr in der Lage, selbst einen Termin beim Zahnarzt zu vereinbaren und diesen auch aufzusuchen. Insgesamt jedoch lasse sich feststellen, dass sich zahnmedizinische Krankheitslasten ins höhere Lebensalter verschieben, stellte Studienleiter Jordan fest. Die älteren Senioren hätten heute einen Mundgesundheitszustand wie die jüngeren Senioren bei der Studie (DMS IV) vor zehn Jahren.

Für die bundesweit größte repräsentative Umfrage zur Mundgesundheit hatte das IDZ zwischen Oktober 2013 und Juli 2014 insgesamt 4600 Deutsche befragt und untersucht. Zusätzlich zu klinischen Daten berücksichtigt die Studie auch soziografische und verhaltensbezogene Einflüsse.

Carolin Stehr

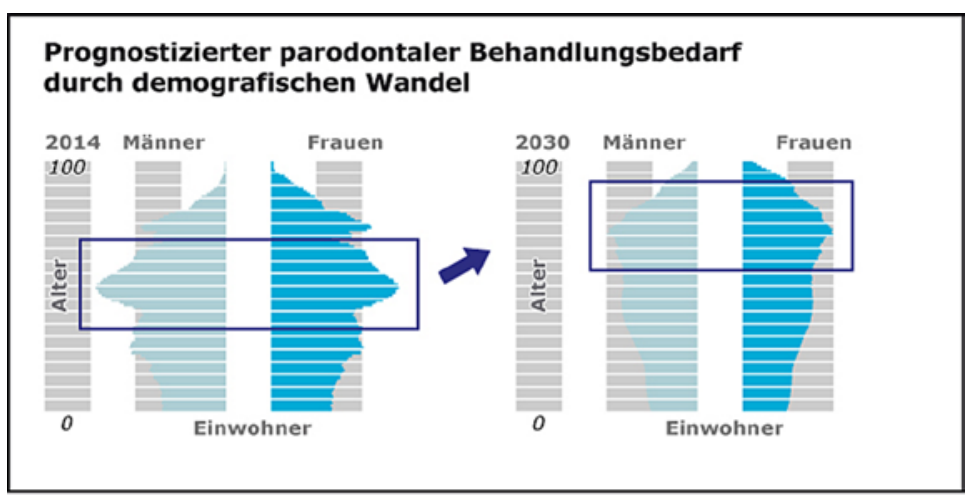

Parodontalerkrankungen sind altersassoziiert. Moderate und schwere Parodontalerkrankungen bei jüngeren Erwachsenen (35- bis 44-Jăhrige) heute: $51,6 \%$ und bei jüngeren Senioren (65- bis 74-Jährige) heute: $64,6 \%$. Im Jahr 2030 werden der Großteil der Bevölkerung Senioren sein. Trotz abnehmender Prăvalenzen ist daher derzeit mit einer Zunahme des parodontalen Behandlungsbedarfs zu rechnen 